\title{
Design and fabrication of dual specimen rotating bending fatigue testing machine
}

\author{
Shreyas $\mathbf{P}^{1}$, Trishul M.A ${ }^{2}$, Chethan Kumar $\mathbf{R}^{3}$, Karthik Babu K. ${ }^{4}$ \\ Asst Prof., Mechanical Engineering Department, CMRIT, (Visvesvaraya Technological University), Bengaluru, India ${ }^{1}$ \\ Assistant Professor, Mechanical Engineering Department, PMCE, (Anna University), Hosur, India ${ }^{2}$ \\ M.Tech Student (Research Scholar), Thermal Power Engineering,The Oxford College of Engineering, Bengaluru,India ${ }^{3}$ \\ Design Engineer, SaiCorp Trailers, Peenya, Bengaluru, India ${ }^{4}$

\begin{abstract}
Testing different materials for their fatigue behaviour has been a major requirement before consider the material for use in any environment with continuous fluctuating load. There has been major evolution in the design of fatigue testing machines for over a century now. But the concept that was lacking in the testing industry was that of a fatigue testing machine that can simultaneously test the fatigue strength of two specimen. In this paper, the attempt to bring this concept to life has been explained. The design of this fatigue testing machine is different from other fatigue testing machines because it can hold two specimens at any given time.
\end{abstract}

Keywords: Fatigue Testing Machine, Moore Test, Rotating Bending.

\section{INTRODUCTION}

Fatigue is the condition whereby a material Crack Growth (Np) -

cracks or fails as a result of repeated (cyclic) stresses Cycles required to grow the crack in a stable manner to a applied below the ultimate strength of the material. Fatigue critical size. Generally controlled by stress level. Since failures often occur quite suddenly with catastrophic most common materials contain flaws, the prediction of (disastrous) results and although most insidious for metals, crack growth is the most studied aspect of fatigue.

polymers and ceramics (except for glasses) are also susceptible to sudden fatigue failures. Fatigue causes brittle like failures even in normally ductile materials with little gross plastic deformation occurring prior to fracture[1]. The process occurs by the initiation and propagation of cracks and, ordinarily, the fracture surface is close to perpendicular to the direction of maximum tensile stress.

Applied stresses may be axial (tension-compression), flexural (bending) or torsional (twisting) in nature. In general there are three possible fluctuating stress-time modes possible. The simplest is completely reversed constant amplitude where the alternating stress varies from a maximum tensile stress to a minimum compressive stress of equal magnitude. The second type, termed repeated constant amplitude[2], occurs when the maxima and minima are asymmetrical relative to the zero stress level. Lastly, the stress level may vary randomly in amplitude and frequency which is merely termed random cycling.

Tensile stresses are normally considered positive and compressive stresses are considered negative. The Fatigue Life (Nf) of a component is defined by the total number of stress cycles required to cause failure. Fatigue Life can be separated into three stages where

$$
\mathbf{N f}=\mathbf{N i}+\mathbf{N p}
$$

Crack Initiation (Ni) -

Cycles required to initiate a crack. Generally results from dislocation pile-ups and/or imperfections such as surface scratches, voids, etc.

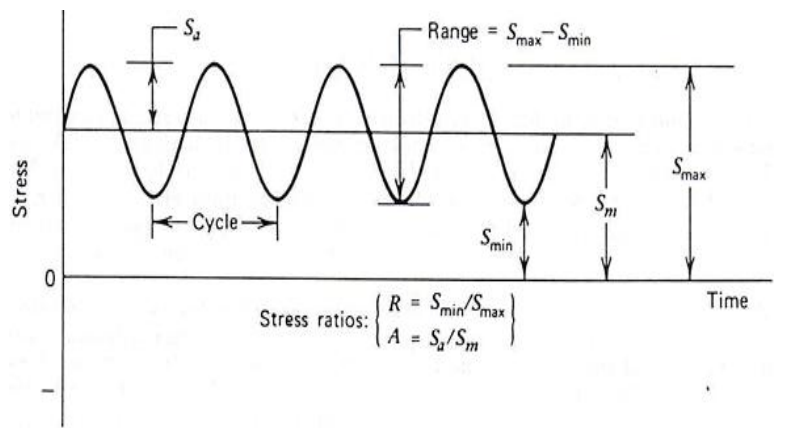

Fig 1.1 Schematic Illustrating Cyclic Loading Parameters

\section{ROTATING BENDING FATIGUE TEST}

Moore test is the standard for rotating bending fatigue test.

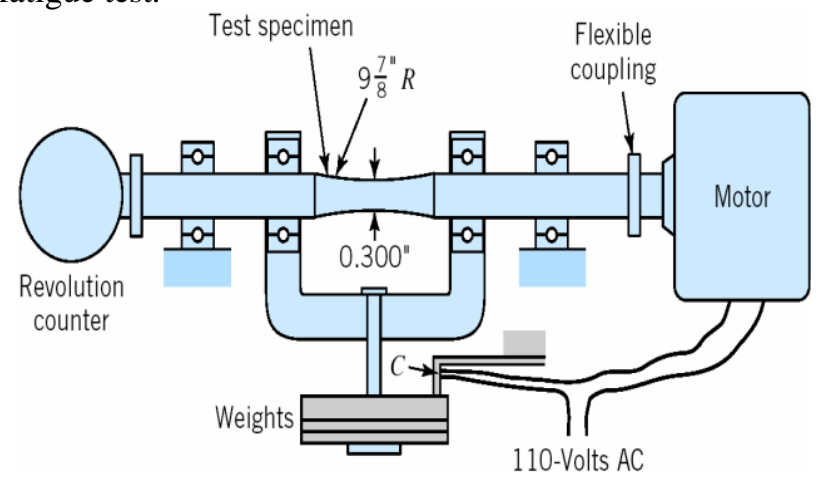

Fig 2.1 Moore Rotating Bending Fatigue Test Apparatus. 
This fatigue testing setup was developed by R.R. FRAME AND SEAT OF MOTOR

Moore[3]. It is used to determine the fatigue strength of a cylindrical specimen to a continuous rotating bending load condition. The machine design is based on the rotating beam principle. The specimen functions as a simple beam symmetrically loaded at two points. When rotated one half revolution, the stresses in the fibres originally below the neutral axis are reversed from tension to compression and vice versa[4]. Upon completing the revolution, the stresses are again reversed so that during one revolution the test specimen passes through a complete cycle of flexural stress (tension and compression).

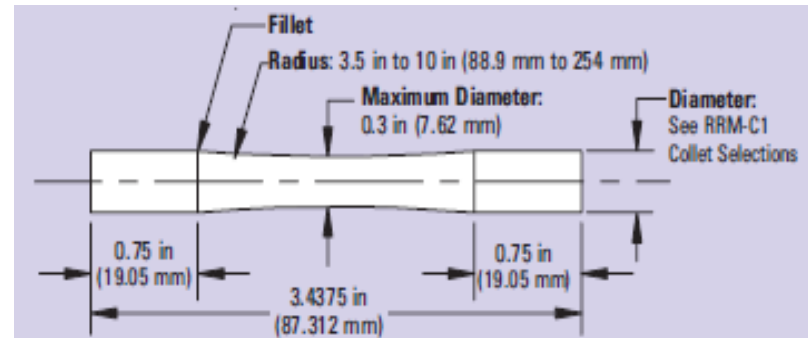

Fig 2.2 Specimen used in Moore fatigue testing.

\section{DESIGN OF THE DUAL SPECIMEN ROTATING BENDING FATIGUE TESTING RIG}

\section{DESIGN THEORY}

The theory governing the design of the fatigue machine is the cantilever loading elastic beam bending principle often referred to as the technical theory of bending. A beam is a relatively long member that can support loads perpendicular to its axis. It can also support applied moments that tend to bend it resulting in the compression of the lower layers of the beam and the extension of the upper layers of the beam. The stress on the beam as a result of the bending is referred to as bending stresses.

The adaptation of this theory is applied in the workings of the cantilever loading type of rotating bending fatigue test which consists in the application of a known constant bending stress (due to a bending moment) to a round specimen on one end which is not hinged while the other extreme end of the specimen is fixed, combined with the rotation of the sample around the bending stress axis until failure occurs. The rotation and simultaneous bending on which the fatigue machine operates ensures that the bending stresses which leads to stretch the upper layers of the specimen and compress the bottom layers as is applicable in stationary beams; is evenly distributed around the entire circumference of the specimen.

\section{MACHINE DESIGN CONSIDERATIONS}

The main parts of the fatigue machine are the electric motor, which gives rotation; two main bearings, which create the supports; proximity sensor, which detects the rotation motion of the shaft and sends counter; digital counter, which takes to failure of the specimen. The various parts/components of the fatigue machine were systematically coupled together through the preparation of design drawings application of the theoretical principles of bending which had been thoroughly studied.
The frames were cut from 40 angle bar of dimension alloy steel of good strength and toughness and welded together to serve as support for the whole set up. A flat flexible metallic plate was cut and attached to a part of the frame to serve as seat for the electric motor. The flexibility is to accommodate ease of adjustment of the electric motor and belt transmitting motion from the electric motor to the shaft.

\section{ASSEMBLY}

The different fabricated and purchased part are then assembled together to form the required setup as shown in the design. This starts by passing the turned shaft through the bearing in the housing forcefully and the shaft is allowed to extend beyond the bearing housing. A pulley is fixed to the electric motor and another to the extended shaft of the fixed bearing housing.

The electric motor is then securely fastened to its seat and properly aligned with the upper pulley. The chuck is then screwed to the threaded mouth of the shaft and the two bearing housing are tightly screwed to the wooden base and properly aligned with each other. Finishing operation involves the addition of body fillers and grinding of all the parts of the machine using emery papers to make sure the parts are smooth. Thereafter, spraying of the machine was performed and the wooden coverings attached.

\section{CONTROL OF THE MACHINE}

The machine is controlled by two switches; one switch turns on the whole system while the other puts on the electric motor which eventually starts the whole experiment.

The magnitude of the load used for testing is predetermined and is applied through the loading arm of the fatigue machine. The bending moment on the specimen and the bending stress are calculated using the relevant relations as discussed in section. The number of cycles to achieve failure is recorded on the digital counter. The electric motor operates at a constant speed of

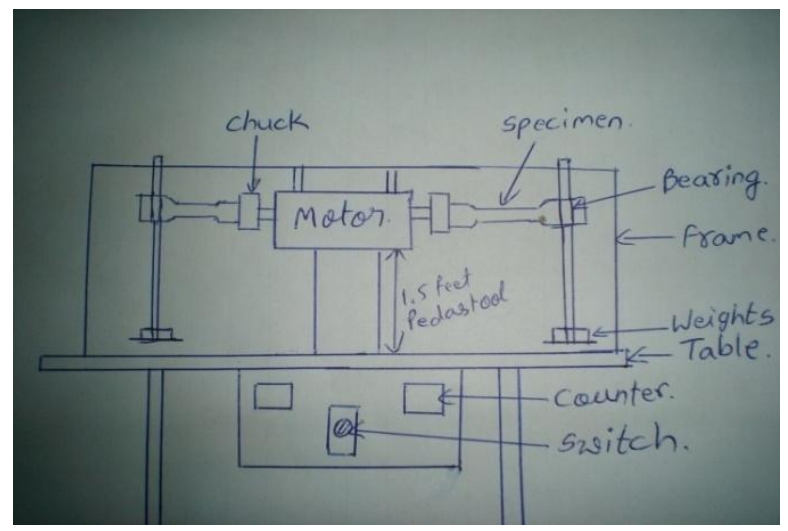

Fig 3.1 Initial drawing of the test rig 


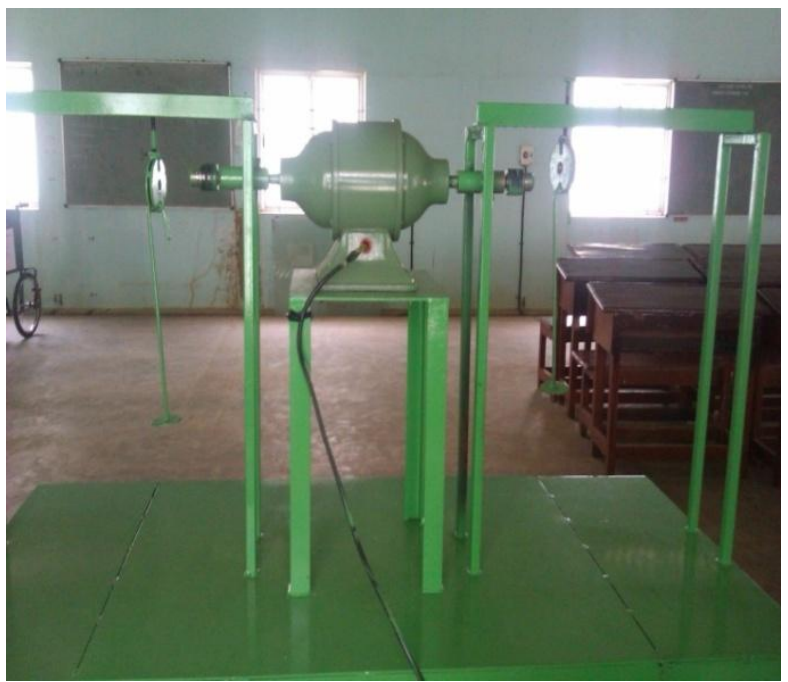

Fig 3.2 Actual fabricated Test Rig

$2920 \mathrm{rev} / \mathrm{min}$ and a frequency of $50 \mathrm{~Hz}$. The revolution counting can be achieved with precision by ensuring that the distance between the proximity sensor and the rotating shaft does not exceed $15 \mathrm{~mm}$. This will imply regular check after each operation of the machine.

\section{ADVANTAGES OF THE DESIGNED TEST RIG}

- $\quad$ Two specimens can be simultaneously tested.

- Uses comparatively less power for testing two specimens.

- $\quad$ The design is unique, original, ergonomic and very sturdy.

- $\quad$ Specimens made of two different materials can be tested simultaneously and a basic comparative study can be made there itself.

- $\quad$ Reduces the number of resources used like just one motor instead of two for two specimens, which makes it environment friendly.

- $\quad$ Takes comparatively less time for fatigue testing of two specimens.

\section{REFERENCES}

[1] John Pruett, David J. Clement, Et. al., Cyclic fatigue testing of nickel-titanium endodontic instrument, JOE, Volume 23 Issue 2 (1997), Pg. 77-85.

[2] Tommy J. George, Et. al., Development of a novel vibration-based fatigue testing methodology, International Journal of Fatigue, Volume 26, Issue 5, May 2004, Pages 477-486.

[3] Stephen D. Cook, Et. al., Fatigue properties of carbon- and porouscoated $\mathrm{Ti}=6 \mathrm{Al}=4 \mathrm{~V}$ alloy, Journal of Biomedical Materials Research, Volume 18, Issue 5, pages 497-512, May/June 1984.

[4] Stephen D. Cook, Et. al., The effect of post-sintering heat treatments on the fatigue properties of porous coated Ti-6Al-4V Alloy, Journal of Biomedical Materials Research, Volume 22, Issue 4, pages 287302, April 1988. 